\title{
ON SOME WEAKER HESITANT FUZZY OPEN SETS
}

\author{
Hariwan Z. IBRAHIM \\ Department of Mathematics, Faculty of Education, University of Zakho, \\ Zakho, IRAQ
}

\begin{abstract}
The purpose of this paper is to define and study some new types of hesitant fuzzy open sets namely, hesitant fuzzy $\alpha$-open, hesitant fuzzy preopen, hesitant fuzzy semiopen, hesitant fuzzy b-open and hesitant fuzzy $\beta$-open in hesitant fuzzy topological space. Some properties and the relationships between these hesitant fuzzy sets are investigated. Furthermore, some relationships between them in hesitant fuzzy subspace are introduced.
\end{abstract}

\section{INTRODUCTION}

Hesitant fuzzy sets are very useful to deal with group decision making problems when experts have a hesitation among several possible memberships for an element to a set. During the evaluating process in practice, however, these possible memberships may be not only crisp values in $[0,1]$, but also interval values. Then hesitant fuzzy set theory has many applications in various fields like decision making problems, decision support systems, clustering algorithms, algebras, etc. After that time, hesitant fuzzy set theory has been developed rapidly by some scholars in theory and practice. In 1965, Zadeh 16 introduced the concept of a fuzzy set as a generalization of a crisp set. Chang [3] defined initially the notion of fuzzy topological spaces. In 2010, Torra 14 introduced the notion of a hesitant fuzzy set as an extension of a fuzzy set. In 2011, Xia and Xu 15 applied a hesitant fuzzy set to decision making by defining "hesitant fuzzy information aggregation". Jun et al. 5 studied hesitant fuzzy bi-ideals in semigroups. Divakaran and John [4] introduced a basic version of hesitant fuzzy rough sets through hesitant fuzzy relations. On the other hand, Jun and Ahn [6] applied hesitant fuzzy sets to BCK/BCI-algebras. Kim et al. 7] gave characterizations of a hesitant fuzzy positive implicative ideal, a hesitant fuzzy implicative ideal, and a hesitant fuzzy commutative ideal, respectively

2020 Mathematics Subject Classification. Primary: 54A05, 03E72; Secondary: 54A40.

Keywords. Hesitant fuzzy sets, hesitant fuzzy topology, hesitant fuzzy interior, hesitant fuzzy closure, hesitant fuzzy subspaces.

๑ hariwan_math@yahoo.com

(D) 0000-0001-9417-2695.

(C)2021 Ankara University Communications Faculty of Sciences University of Ankara Series A1 Mathematics and Statistics 
in BCK-algebras. Recently, Lee and Hur [10] defined a hesitant fuzzy topology and introduced the concepts of a hesitant fuzzy neighborhood, closure, interior, hesitant fuzzy subspace and obtained some of their properties. Also, they defined a hesitant fuzzy continuous mapping and investigated some of its properties. In 1965, Njastad 13 defined the class of $\alpha$-open sets in topological spaces. In 1982, Mashhour et al 12 introduced the concept of preopen sets. The study of semiopen sets and their properties was initiated by Levine [11]. In 1996, Andrijevic' 2 introduced and studied a class of generalized open sets in a topological space called b-open sets, this class of sets contained in the class of $\beta$-open sets [1] and contains all semiopen sets and all preopen sets.

\section{HESITANT FUZZY OPEN SETS}

Definition 1. [14] Let $X$ be a reference set, and $P[0,1]$ denote the power set of $[0,1]$. Then, a mapping $h: X \rightarrow P[0,1]$ is called a hesitant fuzzy set in $X$.

The hesitant fuzzy empty (resp. whole) set, denoted by $h^{0}\left(\right.$ resp. $\left.h^{1}\right)$, is a hesitant fuzzy set in $X$ defined as $h^{0}(x)=\phi\left(\right.$ resp. $\left.h^{1}(x)=[0,1]\right)$, for each $x \in X$. Especially, we will denote the set of all hesitant fuzzy sets in $X$ as $H S(X)[8]$.

Definition 2. Assume that $X$ is a nonempty set and $h, h_{i} \in H S(X)$ for $i$ belong to the set of natural numbers $N$. Then,

(1) $h_{1}$ is a subset of $h_{2}$, denoted by $h_{1} \subseteq h_{2}$, if $h_{1}(x) \subseteq h_{2}(x)$, for each $x \in X$ [4].

(2) $h_{1}$ is equal to $h_{2}$, denoted by $h_{1}=h_{2}$, if $h_{1}(x) \subseteq h_{2}(x)$ and $h_{2}(x) \subseteq h_{1}(x)$ [4].

(3) the intersection of $h_{1}$ and $h_{2}$, denoted by $h_{1} \widetilde{\cap} h_{2}$, is a hesitant fuzzy set in $X$ defined as follows: for each $x \in X$, $\left(h_{1} \widetilde{\cap} h_{2}\right)(x)=h_{1}(x) \cap h_{2}(x)[8]$.

(4) the union of $h_{1}$ and $h_{2}$, denoted by $h_{1} \widetilde{\cup} h_{2}$, is a hesitant fuzzy set in $X$ defined as follows: for each $x \in X$, $\left(h_{1} \widetilde{\cup} h_{2}\right)(x)=h_{1}(x) \cup h_{2}(x)[8]$.

(5) the complement of $h$, denoted by $h^{c}$, is a hesitant fuzzy set in $X$ defined as: for each $x \in X$,

$$
h^{c}(x)=h(x)^{c}=[0,1] \backslash h(x)\lceil 8] .
$$

(6) the intersection of $\left\{h_{i}\right\}_{i \in N}$, denoted by $\widetilde{\bigcap}_{i \in N} h_{i}$, is a hesitant fuzzy set in $X$ defined as follows: for each $x \in X$,

$$
\left(\widetilde{\bigcap}_{i \in N} h_{i}\right)(x)=\bigcap_{i \in N} h_{i}(x)[8] \text {. }
$$

(7) the union of $\left\{h_{i}\right\}_{i \in N}$, denoted by $\widetilde{\cup}_{i \in N} h_{i}$, is a hesitant fuzzy set in $X$ defined as follows: for each $x \in X$,

$$
\left(\widetilde{\bigcup}_{i \in N} h_{i}\right)(x)=\bigcup_{i \in N} h_{i}(x)[8] \text {. }
$$

Definition 3. [9] Let $h \in H S(X)$. Then, $h$ is called a hesitant fuzzy point with the support $x \in X$ and the value $\delta$, denoted by $x_{\delta}$, if $x_{\delta}: X \rightarrow P[0,1]$ is the mapping 
given by: for each $y \in X$,

$$
x_{\delta}(y)= \begin{cases}\delta \subseteq[0,1] & \text { if } y=x \\ \phi & \text { otherwise. }\end{cases}
$$

In particular, $H_{P}(X)$ is called the set of all hesitant fuzzy points in $X$. If $\delta \subseteq h(x)$, then $x_{\delta}$ is said to belong to $h$, denoted by $x_{\delta} \in h$. It is obvious that $h=\widetilde{U}_{x_{\delta} \in h} x_{\delta}$.

Definition 4. [10] Let $X$ be a nonempty set, and $\tau \subseteq H S(X)$. Then, $\tau$ is called a hesitant topology $(H F T)$ on $X$, if it satisfies the following axioms:

(1) $h^{0}, h^{1} \in \tau$.

(2) For any $h_{1}, h_{2} \in \tau$, we have $h_{1} \widetilde{\cap} h_{2} \in \tau$.

(3) For each $h_{i} \in \tau$, we have $\widetilde{\cup}_{i \in N} h_{i} \in \tau$.

The pair $(X, \tau)$ is called a hesitant fuzzy topological space. Each member of $\tau$ is called a hesitant fuzzy open set (HFOS) in X. A hesitant fuzzy set $h$ in $X$ is called a hesitant fuzzy closed set (HFCS) in $(X, \tau)$, if $h^{c} \in \tau$. The set of all hesitant fuzzy closed sets is denoted by $H F C(X)$.

Definition 5. [10] Let $(X, \tau)$ be a hesitant fuzzy topological space, and $h_{A} \in$ $H S(X)$. Then:

(1) $\operatorname{int}_{H}\left(h_{A}\right)=\widetilde{\bigcup}\left\{h_{U} \in \tau: h_{U} \subseteq h_{A}\right\}$.

(2) $c l_{H}\left(h_{A}\right)=\widetilde{\bigcap}\left\{h_{F} \in H F C(X): h_{A} \subseteq h_{F}\right\}$.

\section{WEAKER HESITANT FUZZY OPEN SETS}

Definition 6. Let $(X, \tau)$ be a hesitant fuzzy topological space. A subset $h$ of $H S(X)$ is called:

(1) hesitant fuzzy $\alpha$-open if $h \subseteq \operatorname{int}_{H}\left(c_{H}\left(\right.\right.$ int $\left.\left._{H}(h)\right)\right)$.

(2) hesitant fuzzy preopen if $h \subseteq$ int $_{H}\left(c l_{H}(h)\right)$.

(3) hesitant fuzzy semiopen if $h \subseteq \mathrm{cl}_{H}\left(\right.$ int $\left._{H}(h)\right)$.

(4) hesitant fuzzy b-open if $h \subseteq \operatorname{int}_{H}\left(c l_{H}(h)\right) \widetilde{\cup} c l_{H}\left(\right.$ int $\left._{H}(h)\right)$.

(5) hesitant fuzzy $\beta$-open if $h \subseteq c_{H}\left(\right.$ int $\left._{H}\left(c l_{H}(h)\right)\right)$.

Theorem 1. Let $(X, \tau)$ be a hesitant fuzzy topological space, then the following statements are hold:

(1) Every hesitant fuzzy open set is hesitant fuzzy $\alpha$-open.

(2) Every hesitant fuzzy $\alpha$-open set is hesitant fuzzy preopen.

(3) Every hesitant fuzzy $\alpha$-open set is hesitant fuzzy semiopen.

(4) Every hesitant fuzzy preopen set is hesitant fuzzy b-open.

(5) Every hesitant fuzzy semiopen set is hesitant fuzzy b-open.

(6) Every hesitant fuzzy b-open set is hesitant fuzzy $\beta$-open.

Proof. (1) If $h_{A}$ is hesitant fuzzy open, then $h_{A}=i n t_{H}\left(h_{A}\right) \subseteq i n t_{H}\left(c l_{H}\left(h_{A}\right)\right)=$ $\operatorname{int}_{H}\left(c l_{H}\left(\operatorname{int}_{H}\left(h_{A}\right)\right)\right)$. Thus, $h_{A}$ is hesitant fuzzy $\alpha$-open. 
(2) If $h_{A}$ is hesitant fuzzy $\alpha$-open, then $h_{A} \subseteq \operatorname{int}_{H}\left(c_{H}\left(i n t_{H}\left(h_{A}\right)\right)\right) \subseteq i n t_{H}\left(c l_{H}\left(h_{A}\right)\right)$. Thus, $h_{A}$ is hesitant fuzzy preopen.

(3) If $h_{A}$ is hesitant fuzzy $\alpha$-open, then $h_{A} \subseteq i n t_{H}\left(c l_{H}\left(i n t_{H}\left(h_{A}\right)\right)\right) \subseteq \operatorname{cl}_{H}\left(i n_{H}\left(h_{A}\right)\right)$. Thus, $h_{A}$ is hesitant fuzzy semiopen.

(4) If $h_{A}$ is hesitant fuzzy preopen, then $h_{A} \subseteq i n t_{H}\left(c l_{H}\left(h_{A}\right)\right) \subseteq i n t_{H}\left(c l_{H}\left(h_{A}\right)\right)$ $\widetilde{U} c l_{H}\left(\operatorname{int}_{H}\left(h_{A}\right)\right)$. Thus, $h_{A}$ is hesitant fuzzy b-open.

(5) If $h_{A}$ is hesitant fuzzy semiopen, then $h_{A} \subseteq c l_{H}\left(\operatorname{int}_{H}\left(h_{A}\right)\right) \subseteq i n t_{H}\left(c l_{H}\left(h_{A}\right)\right)$ $\widetilde{U} c l_{H}\left(\operatorname{int}_{H}\left(h_{A}\right)\right)$. Thus, $h_{A}$ is hesitant fuzzy b-open.

(6) If $h_{A}$ is hesitant fuzzy b-open, then $h_{A} \subseteq i n t_{H}\left(c l_{H}\left(h_{A}\right)\right) \widetilde{\cup} c l_{H}\left(i n t_{H}\left(h_{A}\right)\right) \subseteq$ $c l_{H}\left(\operatorname{int}_{H}\left(c l_{H}\left(h_{A}\right)\right)\right) \widetilde{\cup} c l_{H}\left(i n t_{H}\left(h_{A}\right)\right)=\operatorname{cl}_{H}\left[i n t_{H}\left(c l_{H}\left(h_{A}\right)\right) \widetilde{\cup} i n t_{H}\left(h_{A}\right)\right] \subseteq$ $c l_{H}\left[\operatorname{int}_{H}\left(c l_{H}\left(h_{A}\right)\right) \tilde{\cup} \operatorname{int}_{H}\left(c l_{H}\left(h_{A}\right)\right)\right]=c_{H}\left(\operatorname{int}_{H}\left(c l_{H}\left(h_{A}\right)\right)\right)$. Thus, $h_{A}$ is hesitant fuzzy $\beta$-open.

Remark 1. The concepts of hesitant fuzzy preopen and hesitant fuzzy semiopen are independent.

Remark 2. The converse of the Theorem 1, need not be true as shown by the following examples.

Example 1. Consider the hesitant fuzzy sets in $X=\{a, b, c\}$ given by:

$h_{1}(a)=[0.7,1], h_{1}(b)=\{0.2,0.5,0.8\}, h_{1}(c)=[0.7,1)$,

$h_{2}(a)=[0.5,1), h_{2}(b)=\{0.2,0.5,0.7\}, h_{2}(c)=(0.7,1]$,

$h_{3}(a)=[0.7,1), h_{3}(b)=\{0.2,0.5\}, h_{3}(c)=(0.7,1)$, and

$h_{4}(a)=[0.5,1], h_{4}(b)=\{0.2,0.5,0.7,0.8\}, h_{4}(c)=[0.7,1]$.

Then, $\tau=\left\{h^{0}, h^{1}, h_{1}, h_{2}, h_{3}, h_{4}\right\}$ a hesitant topology on $X$. If $h_{A}$ is the hesitant fuzzy set in $X$ given by:

(1) $h_{A}(a)=[0.6,1], h_{A}(b)=\{0.2,0.5,0.6,0.8,0.9\}, h_{A}(c)=[0.3,1)$,

then $h_{A}$ is hesitant fuzzy $\alpha$-open but $h_{A}$ is not hesitant fuzzy open.

(2) $h_{A}(a)=[0,1], h_{A}(b)=\phi, h_{A}(c)=\phi$,

then $h_{A}$ is both hesitant fuzzy preopen and hesitant fuzzy b-open but $h_{A}$ is neither hesitant fuzzy $\alpha$-open nor hesitant fuzzy semiopen.

Example 2. Consider the hesitant fuzzy sets in $X=\{a, b, c\}$ given by:

$h_{1}(a)=\{0.4\}, h_{1}(b)=\{0.1\}, h_{1}(c)=\{0.8\}$,

$h_{2}(a)=\{0.3\}, h_{2}(b)=\{0.2\}, h_{2}(c)=\{0.7\}$, and

$h_{3}(a)=\{0.3,0.4\}, h_{3}(b)=\{0.1,0.2\}, h_{3}(c)=\{0.7,0.8\}$.

Then, $\tau=\left\{h^{0}, h^{1}, h_{1}, h_{2}, h_{3}\right\}$ a hesitant topology on $X$. If $h_{A}$ is the hesitant fuzzy set in $X$ given by:

$h_{A}(a)=\{0.4,0.6\}, h_{A}(b)=\{0.1,0.6\}, h_{A}(c)=\{0.6,0.8\}$,

then $h_{A}$ is both hesitant fuzzy semiopen and hesitant fuzzy b-open but $A$ is neither hesitant fuzzy $\alpha$-open nor hesitant fuzzy preopen.

Example 3. Consider the hesitant fuzzy sets in $X=\{a\}$ given by: $h_{1}(a)=\{0.1\}$, 
$h_{2}(a)=\{0.2\}$,

$h_{3}(a)=\{0.1,0.2\}$,

$h_{4}(a)=\{0.3,0.4\}$,

$h_{5}(a)=\{0.1,0.3,0.4\}$,

$h_{6}(a)=\{0.2,0.3,0.4\}$,

$h_{7}(a)=\{0.1,0.2,0.3,0.4\}$,

$h_{8}(a)=\{0.1,0.3,0.4,0.5\}$, and

$h_{9}(a)=\{0.1,0.2,0.3,0.4,0.5\}$.

Then, $\tau=\left\{h^{0}, h^{1}, h_{1}, h_{2}, h_{3}, h_{4}, h_{5}, h_{6}, h_{7}, h_{8}, h_{9}\right\}$ is a hesitant topology on $X$. If

$h_{A}$ is the hesitant fuzzy set in $X$ given by:

$h_{A}(a)=\{0.2,0.3,0.5\}$,

then $h_{A}$ is hesitant fuzzy $\beta$-open but $A$ is not hesitant fuzzy b-open.

Remark 3. From Theorem 1, we obtain the following diagram of implications:

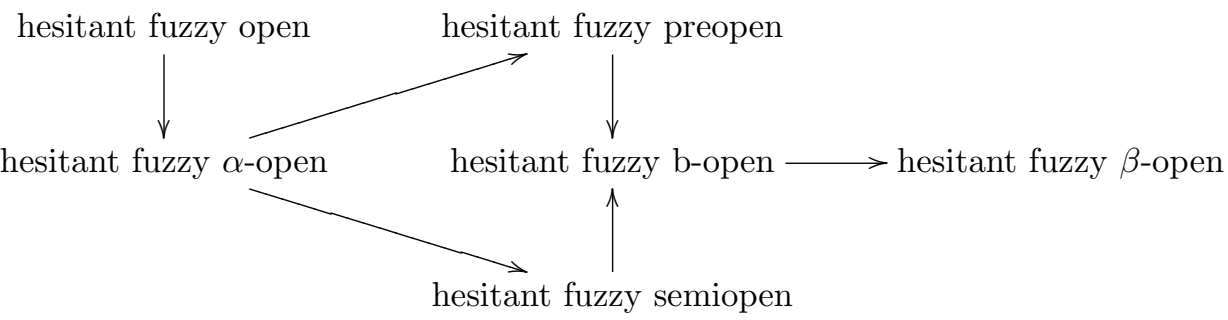

Theorem 2. Let $(X, \tau)$ be a hesitant fuzzy topological space and $h_{A} \in H S(X)$. Then:

(1) $c l_{H}\left(h_{A}\right) \widetilde{\cap} h_{G} \subseteq c l_{H}\left(h_{A} \widetilde{\cap} h_{G}\right)$, for every hesitant fuzzy open set $h_{G}$.

(2) $\operatorname{int}_{H}\left(h_{A} \widetilde{\cup} h_{F}\right) \subseteq \operatorname{int}_{H}\left(h_{A}\right) \widetilde{\cup} h_{F}$, for every hesitant fuzzy closed set $h_{F}$.

Proof. (1) Let $x_{\delta} \in c l_{H}\left(h_{A}\right) \widetilde{\cap} h_{G}$, then $x_{\delta} \in c l_{H}\left(h_{A}\right)$ and $x_{\delta} \in h_{G}$. If $h_{V}$ is a hesitant fuzzy open set containing $x_{\delta}$, then, $h_{V} \widetilde{\cap} h_{G}$ is also hesitant fuzzy open set containing $x_{\delta}$. Since $x_{\delta} \in c l_{H}\left(h_{A}\right)$ implies $\left(h_{V} \widetilde{\cap} h_{G}\right) \widetilde{\cap} h_{A} \neq h^{0}$ and hence $h_{V} \widetilde{\cap}\left(h_{G} \widetilde{\cap} h_{A}\right) \neq h^{0}$. This is true for every $h_{V}$ containing $x_{\delta}$, so $x_{\delta} \in c l_{H}\left(h_{G} \widetilde{\cap} h_{A}\right)$. Therefore hesitant fuzzy $c l\left(h_{A}\right) \widetilde{\cap} h_{G} \subseteq c l_{H}\left(h_{A} \widetilde{\cap} h_{G}\right)$.

(2) Follows from (1) and so it is obvious.

Theorem 3. If $\left\{h_{i}: i \in \mathbf{N}\right\}$ is a collection of hesitant fuzzy b-open (resp. hesitant fuzzy $\alpha$-open, hesitant fuzzy preopen, hesitant fuzzy semiopen and hesitant fuzzy $\beta$-open) sets of a hesitant fuzzy topological space $(X, \tau)$, then $\widetilde{\cup}_{i \in \mathbf{N}} h_{i}$ is a hesitant fuzzy b-open (resp. hesitant fuzzy $\alpha$-open, hesitant fuzzy preopen, hesitant fuzzy semiopen and hesitant fuzzy $\beta$-open) set.

Proof. We prove only the first case since the other cases are similarly shown. Since $h_{i} \subseteq \operatorname{int}_{H}\left(c l_{H}\left(h_{i}\right)\right) \widetilde{\cup} c l_{H}\left(i n t_{H}\left(h_{i}\right)\right)$ for every $i \in \mathbf{N}$, we have

$$
\widetilde{\cup}_{i \in \mathbf{N}} h_{i} \subseteq \widetilde{\cup}_{i \in \mathbf{N}}\left[i n t_{H}\left(c l_{H}\left(h_{i}\right)\right) \widetilde{\cup} c l_{H}\left(i n t_{H}\left(h_{i}\right)\right)\right]
$$




$$
\begin{aligned}
& \subseteq\left[\widetilde{\cup}_{i \in \mathbf{N}} i n t_{H}\left(c l_{H}\left(h_{i}\right)\right)\right] \widetilde{\cup}\left[\widetilde{\cup}_{i \in \mathbf{N}} c l_{H}\left(i n t_{H}\left(h_{i}\right)\right)\right] \\
& \subseteq\left[i n t_{H}\left(\widetilde{\cup}_{i \in \mathbf{N}} c l_{H}\left(h_{i}\right)\right)\right] \widetilde{\cup}\left[c l_{H}\left(\widetilde{\cup}_{i \in \mathbf{N}} i n t_{H}\left(h_{i}\right)\right)\right] \\
& \subseteq\left[\operatorname{int}_{H}\left(c l_{H}\left(\widetilde{\cup}_{i \in \mathbf{N}} h_{i}\right)\right)\right] \widetilde{\cup}\left[c l_{H}\left(\operatorname{int}_{H}\left(\widetilde{\cup}_{i \in \mathbf{N}} h_{i}\right)\right)\right] .
\end{aligned}
$$

Therefore, $\widetilde{\cup}_{i \in \mathbf{N}} h_{i}$ is hesitant fuzzy b-open.

Theorem 4. Let $(X, \tau)$ be a hesitant fuzzy topological space, $h_{U} \in \tau$ and $h_{A} \in$ $H S(X)$.

(1) If $h_{A}$ is hesitant fuzzy preopen, then $h_{U} \widetilde{\cap} h_{A}$ is hesitant fuzzy preopen.

(2) If $h_{A}$ is hesitant fuzzy semiopen, then $h_{U} \widetilde{\cap} h_{A}$ is hesitant fuzzy semiopen.

Proof. (1) Since $h_{A}$ is hesitant fuzzy preopen and $h_{U}$ is hesitant fuzzy open, then, $h_{A} \subseteq \operatorname{int}_{H}\left(c l_{H}\left(h_{A}\right)\right)$ and $\operatorname{int}_{H}\left(h_{U}\right)=h_{U}$ and so by Theorem 2 (1), $h_{U} \widetilde{\cap} h_{A} \subseteq \operatorname{int}_{H}\left(h_{U}\right) \widetilde{\cap} i n t_{H}\left(c l_{H}\left(h_{A}\right)\right)=i n t_{H}\left(h_{U} \widetilde{\cap} c l_{H}\left(h_{A}\right)\right) \subseteq i n t_{H}\left(c l_{H}\left(h_{U} \widetilde{\cap} h_{A}\right)\right)$. Therefore, $h_{U} \widetilde{\cap} h_{A}$ is hesitant fuzzy preopen.

(2) Since $h_{A}$ is hesitant fuzzy semiopen, then by Theorem 2 (1), $h_{U} \widetilde{\cap} h_{A} \subseteq$ $h_{U} \widetilde{\cap} c l_{H}\left(i n_{H}\left(h_{A}\right)\right) \subseteq c l_{H}\left(h_{U} \widetilde{\cap} i n_{H}\left(h_{A}\right)\right)=c l_{H}\left(i n_{H}\left(h_{U}\right) \widetilde{\cap} i n_{H}\left(h_{A}\right)\right)=$ $c l_{H}\left(i n_{H}\left(h_{U} \widetilde{\cap} h_{A}\right)\right)$. Therefore, $h_{U} \widetilde{\cap} h_{A}$ is hesitant fuzzy semiopen.

Theorem 5. Let $(X, \tau)$ be a hesitant fuzzy topological space, $h_{U} \in \tau$ and $h_{A} \in$ $H S(X)$. If $h_{A}$ is hesitant fuzzy $\beta$-open, then $h_{U} \widetilde{\cap} h_{A}$ is hesitant fuzzy $\beta$-open.

Proof. Since $h_{A}$ is hesitant fuzzy $\beta$-open, then

$$
\begin{gathered}
h_{U} \widetilde{\cap} h_{A} \subseteq h_{U} \widetilde{\cap} c l_{H}\left(\operatorname{int}_{H}\left(c l_{H}\left(h_{A}\right)\right)\right) \\
\subseteq c l_{H}\left(h_{U} \widetilde{\cap} \operatorname{int}_{H}\left(c l_{H}\left(h_{A}\right)\right)\right) \\
=c l_{H}\left(\operatorname{int}_{H}\left(h_{U}\right) \widetilde{\cap} \operatorname{int}_{H}\left(c l_{H}\left(h_{A}\right)\right)\right) \\
=c l_{H}\left(\operatorname{int}_{H}\left(h_{U} \widetilde{\cap} c l_{H}\left(h_{A}\right)\right)\right) \\
\subseteq c l_{H}\left(\operatorname{int}_{H}\left(c l_{H}\left(h_{U} \widetilde{\cap} h_{A}\right)\right)\right) .
\end{gathered}
$$

This shows that $h_{U} \widetilde{\cap} h_{A}$ is hesitant fuzzy $\beta$-open.

Theorem 6. Let $(X, \tau)$ be a hesitant fuzzy topological space, $h_{U} \in \tau$ and $h_{A} \in$ $H S(X)$. If $h_{A}$ is hesitant fuzzy b-open, then $h_{U} \widetilde{\cap} h_{A}$ is hesitant fuzzy b-open.

Proof. Since $h_{A}$ is hesitant fuzzy b-open, then

$$
\begin{gathered}
h_{U} \widetilde{\cap} h_{A} \subseteq h_{U} \widetilde{\cap}\left[i n t_{H}\left(c l_{H}\left(h_{A}\right)\right) \widetilde{\cup} c l_{H}\left(i n t_{H}\left(h_{A}\right)\right)\right] \\
=\left[h_{U} \widetilde{\cap} i n t_{H}\left(c l_{H}\left(h_{A}\right)\right)\right] \widetilde{\cup}\left[h_{U} \widetilde{\cap} c l_{H}\left(i n t_{H}\left(h_{A}\right)\right)\right] \\
=\left[i n t_{H}\left(h_{U}\right) \widetilde{\cap} i n t_{H}\left(c l_{H}\left(h_{A}\right)\right)\right] \widetilde{\cup}\left[h_{U} \widetilde{\cap} c l_{H}\left(\operatorname{int}_{H}\left(h_{A}\right)\right)\right] \\
\subseteq\left[\operatorname{int}_{H}\left(h_{U} \widetilde{\cap} c l_{H}\left(h_{A}\right)\right)\right] \widetilde{\cup}\left[c l_{H}\left(h_{U} \widetilde{\cap} \operatorname{int}_{H}\left(h_{A}\right)\right)\right]
\end{gathered}
$$




$$
\subseteq\left[\operatorname{int}_{H}\left(c l_{H}\left(h_{U} \widetilde{\cap} h_{A}\right)\right)\right] \widetilde{\cup}\left[c_{H}\left(i n t_{H}\left(h_{U} \widetilde{\cap} h_{A}\right)\right)\right] .
$$

This shows that $h_{U} \widetilde{\cap} h_{A}$ is hesitant fuzzy b-open.

Remark 4. We note that the intersection of two hesitant fuzzy preopen (resp. hesitant fuzzy semiopen, hesitant fuzzy b-open and hesitant fuzzy $\beta$-open) sets need not be hesitant fuzzy preopen (resp. hesitant fuzzy semiopen, hesitant fuzzy b-open and hesitant fuzzy $\beta$-open) as can be seen from the following examples:

Example 4. Consider the hesitant fuzzy sets in $X=\{a\}$ given by $h(a)=\{0.3,0.6\}$. Then, $\tau=\left\{h^{0}, h^{1}, h\right\}$ is a hesitant topology on $X$. If $h_{A}(a)=\{0.1,0.3\}$ and $h_{B}(a)=\{0.1,0.6\}$, then $h_{A}$ and $h_{B}$ are hesitant fuzzy preopen (resp. hesitant fuzzy b-open and hesitant fuzzy $\beta$-open), but $h_{A} \widetilde{\cap} h_{B}=\{0.1\}=h_{C}$ which is not hesitant fuzzy preopen (resp. hesitant fuzzy b-open and hesitant fuzzy $\beta$-open).

Example 5. From Example 0 , if $h_{A}$ is the hesitant fuzzy set in $X$ given by:

$h_{A}(a)=\{0.4,0.6\}, h_{A}(b)=\{0.1,0.6\}, h_{A}(c)=\{0.6,0.8\}$,

and $h_{B}$ is the hesitant fuzzy set in $X$ given by:

$h_{B}(a)=\{0.3,0.6\}, h_{B}(b)=\{0.2,0.6\}, h_{B}(c)=\{0.7,0.9\}$,

then $h_{A}$ and $h_{B}$ are hesitant fuzzy semiopen, but $h_{A} \widetilde{\cap} h_{B}=h_{C}$ which is not hesitant fuzzy semiopen, where $h_{C}$ is the hesitant fuzzy set in $X$ given by:

$h_{C}(a)=\{0.6\}, h_{C}(b)=\{0.6\}, h_{C}(c)=\phi$.

Remark 5. From Remark 4, we notice that the family of all hesitant fuzzy preopen (resp. hesitant fuzzy semiopen, hesitant fuzzy b-open and hesitant fuzzy $\beta$-open) sets need not be a topology in general.

Theorem 7. Let $(X, \tau)$ be a hesitant fuzzy topological space. If $h_{A}$ and $h_{B}$ are hesitant fuzzy $\alpha$-open, then $h_{B} \widetilde{\cap} h_{A}$ is also hesitant fuzzy $\alpha$-open.

Proof. Since $h_{A}$ and $h_{B}$ are hesitant fuzzy $\alpha$-open, then

$$
\begin{gathered}
h_{B} \widetilde{\cap} h_{A} \subseteq \operatorname{int}_{H}\left(c l_{H}\left(i n t_{H}\left(h_{B}\right)\right)\right) \widetilde{\cap} i n t_{H}\left(c l_{H}\left(i n t_{H}\left(h_{A}\right)\right)\right) \\
\subseteq i n t_{H}\left[c l_{H}\left(i n t_{H}\left(h_{B}\right)\right) \widetilde{n} i n t_{H}\left(c l_{H}\left(i n t_{H}\left(h_{A}\right)\right)\right)\right] \\
\subseteq i n t_{H} c l_{H}\left[i n t_{H}\left(h_{B}\right) \widetilde{\cap} i n t_{H}\left(c l_{H}\left(i n t_{H}\left(h_{A}\right)\right)\right)\right] \\
\subseteq \operatorname{int}_{H} c l_{H}\left[i n t_{H}\left(h_{B}\right) \widetilde{\cap} c l_{H}\left(i n t_{H}\left(h_{A}\right)\right)\right] \\
\subseteq i n t_{H} c l_{H} c l_{H}\left[i n t_{H}\left(h_{B}\right) \widetilde{n i n t} t_{H}\left(h_{A}\right)\right] \\
\subseteq \operatorname{int}_{H} c l_{H} \operatorname{int}_{H}\left(h_{B} \widetilde{\cap} h_{A}\right) .
\end{gathered}
$$

Thus, $h_{B} \widetilde{\cap} h_{A}$ is hesitant fuzzy $\alpha$-open.

Remark 6. From the Theorems 3 and 7 , we notice that the family of all hesitant fuzzy $\alpha$-open is a topology. 
Theorem 8. Let $(X, \tau)$ be a hesitant fuzzy topological space and $h_{A} \in H S(X)$. If $h_{A}$ is both hesitant fuzzy semiopen and hesitant fuzzy preopen, then $h_{A}$ is hesitant fuzzy $\alpha$-open.

Proof. By assumption, $h_{A} \subseteq \operatorname{cl}_{H}\left(\operatorname{int}_{H}\left(h_{A}\right)\right)$ and $h_{A} \subseteq i n t_{H}\left(c_{H}\left(h_{A}\right)\right)$. Then, $h_{A} \subseteq$ $\operatorname{int}_{H}\left(c l_{H}\left(h_{A}\right)\right) \subseteq \operatorname{int}_{H}\left(c l_{H}\left(c l_{H}\left(\operatorname{int}_{H}\left(h_{A}\right)\right)\right)\right)=\operatorname{int}_{H}\left(c l_{H}\left(\operatorname{int}_{H}\left(h_{A}\right)\right)\right)$. Therefore, $h_{A}$ is hesitant fuzzy $\alpha$-open.

Theorem 9. Let $(X, \tau)$ be a hesitant fuzzy topological space and $h_{A}$ be hesitant fuzzy $\alpha$-open.

(1) If $h_{B}$ is hesitant fuzzy semiopen, then $h_{A} \widetilde{\cap} h_{B}$ is hesitant fuzzy semiopen.

(2) If $h_{B}$ is hesitant fuzzy preopen, then $h_{A} \widetilde{\cap} h_{B}$ is hesitant fuzzy preopen.

Proof. (1) By assumption, $h_{A} \subseteq i n t_{H}\left(\operatorname{cl}_{H}\left(\operatorname{int}_{H}\left(h_{A}\right)\right)\right)$ and $h_{B} \subseteq c l_{H}\left(\operatorname{int}_{H}\left(h_{B}\right)\right)$, then by Theorem 2 (1), we have that

$$
\begin{gathered}
h_{A} \widetilde{\cap} h_{B} \subseteq \operatorname{int}_{H}\left(c l_{H}\left(\operatorname{int}_{H}\left(h_{A}\right)\right)\right) \widetilde{\cap} c l_{H}\left(\operatorname{int}_{H}\left(h_{B}\right)\right) \\
\subseteq c l_{H}\left[i n t_{H}\left(c l_{H}\left(\operatorname{int}_{H}\left(h_{A}\right)\right)\right) \widetilde{\cap} \operatorname{int}_{H}\left(h_{B}\right)\right] \\
\subseteq c l_{H}\left[c l_{H}\left(i n t_{H}\left(h_{A}\right)\right) \widetilde{\cap} i n t_{H}\left(h_{B}\right)\right] \\
\subseteq c l_{H}\left[c l_{H}\left[\operatorname{int}_{H}\left(h_{A}\right) \widetilde{\cap} i n t_{H}\left(h_{B}\right)\right]\right] \\
=c l_{H}\left(\operatorname{int}_{H}\left(h_{A} \widetilde{\cap} h_{B}\right)\right) .
\end{gathered}
$$

Therefore, $h_{A} \widetilde{\cap} h_{B}$ is hesitant fuzzy semiopen.

(2) By assumption, $h_{A} \subseteq i n t_{H}\left(\operatorname{cl}_{H}\left(i n t_{H}\left(h_{A}\right)\right)\right)$ and $h_{B} \subseteq i n t_{H}\left(c l_{H}\left(h_{B}\right)\right)$, then

$$
\begin{gathered}
h_{A} \widetilde{\cap} h_{B} \subseteq \operatorname{int}_{H}\left(c l_{H}\left(\operatorname{int}_{H}\left(h_{A}\right)\right)\right) \tilde{\cap} \operatorname{int}_{H}\left(c l_{H}\left(h_{B}\right)\right) \\
=\operatorname{int}_{H}\left[\operatorname{int}_{H}\left(c l_{H}\left(\operatorname{int}_{H}\left(h_{A}\right)\right)\right) \widetilde{\cap} \operatorname{int}_{H}\left(c l_{H}\left(h_{B}\right)\right)\right] \\
\subseteq \operatorname{int}_{H}\left[c l_{H}\left(\operatorname{int}_{H}\left(h_{A}\right)\right) \widetilde{\cap} \operatorname{int}_{H}\left(c l_{H}\left(h_{B}\right)\right)\right] \\
\subseteq \operatorname{int}_{H}\left[c_{H}\left[i n t_{H}\left(h_{A}\right) \widetilde{\cap} \operatorname{int}_{H}\left(c l_{H}\left(h_{B}\right)\right)\right]\right] \\
\subseteq \operatorname{int}_{H}\left[c l_{H}\left[i n t_{H}\left(h_{A}\right) \widetilde{n} c l_{H}\left(h_{B}\right)\right]\right] \\
\subseteq \operatorname{int}_{H}\left[c l_{H}\left[c l_{H}\left[i n t_{H}\left(h_{A}\right) \widetilde{\cap} h_{B}\right]\right]\right] \\
\subseteq \operatorname{int}_{H}\left(c l_{H}\left(c l_{H}\left(h_{A} \widetilde{\cap} h_{B}\right)\right)\right) \\
=\operatorname{int}_{H}\left(c l_{H}\left(h_{A} \widetilde{\cap} h_{B}\right)\right) .
\end{gathered}
$$

Therefore, $h_{A} \widetilde{\cap} h_{B}$ is hesitant fuzzy preopen.

Theorem 10. Let $(X, \tau)$ be a hesitant fuzzy topological space. If $h_{A}$ is hesitant fuzzy preopen and $h_{B}$ is hesitant fuzzy semiopen, then $h_{A} \widetilde{\cap} h_{B}$ is hesitant fuzzy $\beta$-open.

Proof. By assumption, $h_{A} \subseteq \operatorname{int}_{H}\left(c_{H}\left(h_{A}\right)\right)$ and $h_{B} \subseteq \operatorname{cl}_{H}\left(i n t_{H}\left(h_{B}\right)\right)$, then by Theorem 2 (1), we have that

$$
\begin{gathered}
h_{A} \widetilde{\cap}_{B} \subseteq i n t_{H}\left(c l_{H}\left(h_{A}\right)\right) \widetilde{\cap} c l_{H}\left(i n t_{H}\left(h_{B}\right)\right) \\
\subseteq c l_{H}\left[i n t_{H}\left(c l_{H}\left(h_{A}\right)\right) \widetilde{\cap} i n t_{H}\left(h_{B}\right)\right]
\end{gathered}
$$




$$
\begin{gathered}
=\operatorname{cl}_{H}\left[\operatorname{int}_{H}\left[\operatorname{int}_{H}\left(c l_{H}\left(h_{A}\right)\right) \widetilde{\cap} \tilde{i n t}_{H}\left(h_{B}\right)\right]\right] \\
\subseteq c l_{H}\left[i n t_{H}\left[c l_{H}\left(h_{A}\right) \widetilde{\cap} i n t_{H}\left(h_{B}\right)\right]\right] \\
\subseteq c l_{H}\left[i n t_{H}\left[c l_{H}\left(h_{A} \widetilde{\cap} i n t_{H}\left(h_{B}\right)\right)\right]\right] \\
\subseteq c_{H}\left(\operatorname{int}_{H}\left(c l_{H}\left(h_{A} \widetilde{\cap} h_{B}\right)\right)\right) .
\end{gathered}
$$

Therefore, $h_{A} \widetilde{\cap} h_{B}$ is hesitant fuzzy $\beta$-open.

Theorem 11. Let $(X, \tau)$ be a hesitant fuzzy topological space and $h_{A}, h_{B} \in$ $H S(X)$. Then,

(1) $h_{A}$ is hesitant fuzzy semiopen if and only if there exists a hesitant fuzzy open set $h_{U}$ such that $h_{U} \subseteq h_{A} \subseteq c l_{H}\left(h_{U}\right)$.

(2) $h_{B}$ is hesitant fuzzy semiopen if $h_{A}$ is hesitant fuzzy semiopen and $h_{A} \subseteq$ $h_{B} \subseteq c l_{H}\left(h_{A}\right)$.

(3) $h_{A}$ is hesitant fuzzy semiopen if and only if $c l_{H}\left(h_{A}\right)=c l_{H}\left(i n t_{H}\left(h_{A}\right)\right)$.

Proof. (1) Let $h_{A}$ be hesitant fuzzy semiopen, then $h_{A} \subseteq \operatorname{cl}_{H}\left(\operatorname{int}_{H}\left(h_{A}\right)\right)$. Take $h_{U}=\operatorname{int}_{H}\left(h_{A}\right)$, then $h_{U}$ is hesitant fuzzy open such that $h_{U}=i n t_{H}\left(h_{A}\right) \subseteq$ $h_{A} \subseteq c l_{H}\left(\operatorname{int}_{H}\left(h_{A}\right)\right)=c l_{H}\left(h_{U}\right)$.

Conversely, since $h_{U} \subseteq h_{A}$ implies that $h_{U}=\operatorname{int}_{H}\left(h_{U}\right) \subseteq i n t_{H}\left(h_{A}\right)$ and so $h_{A} \subseteq c l_{H}\left(h_{U}\right)=c l_{H}\left(\operatorname{int}_{H}\left(h_{U}\right)\right) \subseteq c l_{H}\left(\operatorname{int}_{H}\left(h_{A}\right)\right)$. Thus, $h_{A}$ is hesitant fuzzy semiopen.

(2) Since $h_{A}$ is hesitant fuzzy semiopen, then by (1) there exists a hesitant fuzzy open set $h_{U}$ such that $h_{U} \subseteq h_{A} \subseteq c l_{H}\left(h_{U}\right)$. Since $h_{A} \subseteq h_{B}$, so $h_{U} \subseteq h_{B}$. But $c l_{H}\left(h_{A}\right) \subseteq c l_{H}\left(h_{U}\right)$, then $h_{B} \subseteq c l_{H}\left(h_{U}\right)$. Hence, $h_{U} \subseteq h_{B} \subseteq c l_{H}\left(h_{U}\right)$. Thus, $h_{B}$ is hesitant fuzzy semiopen.

(3) Let $h_{A}$ be hesitant fuzzy semiopen, then $h_{A} \subseteq \operatorname{cl}_{H}\left(\operatorname{int}_{H}\left(h_{A}\right)\right)$ which implies that $c l_{H}\left(h_{A}\right) \subseteq c l_{H}\left(i_{n} t_{H}\left(h_{A}\right)\right) \subseteq c l_{H}\left(h_{A}\right)$ and hence $c l_{H}\left(h_{A}\right)=$ $c l_{H}\left(\operatorname{int}_{H}\left(h_{A}\right)\right)$.

Conversely, since by Theorem 1. $\operatorname{int}_{H}\left(h_{A}\right)$ is hesitant fuzzy semiopen such that $\operatorname{int}_{H}\left(h_{A}\right) \subseteq h_{A} \subseteq c l_{H}\left(h_{A}\right)=c_{H}\left(i n t_{H}\left(h_{A}\right)\right)$ and therefore $h_{A}$ is hesitant fuzzy semiopen.

Definition 7. [10] Let $(X, \tau)$ be a hesitant fuzzy topological space and $h \in H S(X)$. Then, the collection $\tau_{h}=\{U \widetilde{\cap} h: U \in \tau\}$ is called a hesitant fuzzy subspace topology or hesitant fuzzy relative topology on $h$. The pair $\left(h, \tau_{h}\right)$ is called a hesitant fuzzy subspace, and each member of $\tau_{h}$ is called a hesitant fuzzy open set in $h$.

Proposition 1. [10] Let $(X, \tau)$ be a hesitant fuzzy topological space, $h, h_{A} \in$ $H S(X)$ and $h_{A} \subseteq h$. Then, $c l_{\tau_{h}}\left(h_{A}\right)=h \widetilde{\cap} c l_{H}\left(h_{A}\right)$, where $c l_{\tau_{h}}\left(h_{A}\right)$ denotes the closure of $h_{A}$ in $\left(h, \tau_{h}\right)$.

Definition 8. Let $(X, \tau)$ be a hesitant fuzzy topological space, $h, h_{A} \in H S(X)$ and $h_{A} \subseteq h$. Then, $\operatorname{int}_{\tau_{h}}\left(h_{A}\right)=\tilde{\bigcup}\left\{h_{U} \in \tau_{h}: h_{U} \subseteq h_{A}\right\}$. 
Theorem 12. Let $(X, \tau)$ be a hesitant fuzzy topological space and $h_{A}, h_{B} \in$ $H S(X)$. If $h_{A}$ is hesitant fuzzy preopen in $X$ and $h_{B}$ is hesitant fuzzy semiopen in $X$, then

(1) $h_{A} \widetilde{\cap} h_{B}$ is hesitant fuzzy semiopen in $h_{A}$.

(2) $h_{A} \widetilde{\cap} h_{B}$ is hesitant fuzzy preopen in $h_{B}$.

Proof. By assumption, $h_{A} \subseteq i n t_{H}\left(c l_{H}\left(h_{A}\right)\right)$ and $h_{B} \subseteq c l_{H}\left(i n t_{H}\left(h_{B}\right)\right)$.

(1) Then,

$$
\begin{aligned}
& h_{A} \widetilde{\cap} h_{B} \subseteq \operatorname{int}_{H}\left(c l_{H}\left(h_{A}\right)\right) \widetilde{\cap} c l_{H}\left(i n t_{H}\left(h_{B}\right)\right) \\
& \subseteq c l_{H}\left[i n t_{H}\left(c l_{H}\left(h_{A}\right)\right) \widetilde{\cap} i n t_{H}\left(h_{B}\right)\right] \\
& \subseteq \\
& \subseteq l_{H}\left[c l_{H}\left(h_{A}\right) \widetilde{\cap} i n t_{H}\left(h_{B}\right)\right] \\
& \subseteq c l_{H}\left[c l_{H}\left[h_{A} \widetilde{\cap} i n t_{H}\left(h_{B}\right)\right]\right] \\
&=c l_{H}\left[h_{A} \widetilde{\cap} i n t_{H}\left(h_{B}\right)\right] .
\end{aligned}
$$

Hence, $h_{A} \widetilde{\cap} h_{B} \subseteq c l_{H}\left(h_{A} \widetilde{\cap} i n t_{H}\left(h_{B}\right)\right)$ and so $h_{A} \widetilde{\cap} h_{B} \subseteq c l_{H}\left(h_{A} \widetilde{\cap} i n t_{H}\left(h_{B}\right)\right) \widetilde{\cap} h_{A}=$ $c l_{\tau_{h_{A}}}\left(h_{A} \widetilde{\cap} i n t_{H}\left(h_{B}\right)\right)$. Since $h_{A} \widetilde{\cap} i n t_{H}\left(h_{B}\right)$ is a hesitant fuzzy open set in $h_{A}$, so $h_{A} \widetilde{\cap} h_{B} \subseteq c l_{\tau_{h_{A}}}\left(h_{A} \widetilde{\cap} i n t_{H}\left(h_{B}\right)\right)=\operatorname{cl}_{\tau_{h_{A}}}\left(\operatorname{int}_{\tau_{h_{A}}}\left(h_{A} \widetilde{\cap} i n t_{H}\left(h_{B}\right)\right)\right) \subseteq$ $c l_{\tau_{h_{A}}}\left(\operatorname{int}_{\tau_{h_{A}}}\left(h_{A} \widetilde{\cap} h_{B}\right)\right)$. Therefore, $h_{A} \widetilde{\cap} h_{B}$ is hesitant fuzzy semiopen in $h_{A}$.

(2) Now,

$$
\begin{gathered}
h_{A} \widetilde{\cap} h_{B} \subseteq i n t_{H}\left(c l_{H}\left(h_{A}\right)\right) \widetilde{\cap} h_{B} \\
=\operatorname{int}_{\tau_{h_{B}}}\left[\operatorname{int}_{H}\left(c l_{H}\left(h_{A}\right)\right) \widetilde{\cap} h_{B}\right] \\
\subseteq \operatorname{int}_{\tau_{h_{B}}}\left[\operatorname{int}_{H}\left(c l_{H}\left(h_{A}\right)\right) \widetilde{\cap} c l_{H}\left(i n t_{H}\left(h_{B}\right)\right)\right] \\
\subseteq \operatorname{int}_{\tau_{h_{B}}}\left[c l_{H}\left[i n t_{H}\left(c l_{H}\left(h_{A}\right)\right) \widetilde{\cap} i n t_{H}\left(h_{B}\right)\right]\right] \\
\subseteq \operatorname{int}_{\tau_{h_{B}}}\left[c l_{H}\left[c l_{H}\left(h_{A}\right) \widetilde{\cap} \operatorname{int}_{H}\left(h_{B}\right)\right]\right] \\
\subseteq \operatorname{int}_{\tau_{h_{B}}}\left[c l_{H}\left[c l_{H}\left[h_{A} \widetilde{\cap} i n t_{H}\left(h_{B}\right)\right]\right]\right] \\
\subseteq \operatorname{int}_{\tau_{h_{B}}}\left[c l_{H}\left[c l_{H}\left[h_{A} \widetilde{\cap} h_{B}\right]\right]\right] \\
=\operatorname{int}_{\tau_{h_{B}}}\left(c l_{H}\left(h_{A} \widetilde{\cap} h_{B}\right)\right) .
\end{gathered}
$$

Since $\operatorname{int}_{\tau_{h_{B}}}\left(c l_{H}\left(h_{A} \widetilde{\cap} h_{B}\right)\right)$ is hesitant fuzzy open in $h_{B}$, then $\operatorname{int}_{\tau_{h_{B}}}\left(c l_{H}\left(h_{A} \widetilde{\cap} h_{B}\right)\right) \widetilde{\cap} h_{B}=i n t_{\tau_{h_{B}}}\left(c l_{H}\left(h_{A} \widetilde{\cap} h_{B}\right) \widetilde{\cap} h_{B}\right)$, and hence $h_{A} \widetilde{\cap} h_{B} \subseteq$ $\operatorname{int}_{\tau_{h_{B}}}\left(c l_{H}\left(h_{A} \widetilde{\cap} h_{B}\right) \widetilde{\cap} h_{B}\right)=i n t_{\tau_{h_{B}}}\left(c l_{\tau_{h_{B}}}\left(h_{A} \widetilde{\cap} h_{B}\right)\right)$.

Therefore, $h_{A} \widetilde{\cap} h_{B}$ is hesitant fuzzy preopen in $h_{B}$.

Theorem 13. Let $(X, \tau)$ be a hesitant fuzzy topological space, $h_{A}, h_{B} \in H S(X)$, $h_{A} \subseteq h_{B}$ and $h_{B}$ be hesitant fuzzy semiopen in $X$. Then, $h_{A}$ is hesitant fuzzy semiopen in $X$ if and only if $h_{A}$ is hesitant fuzzy semiopen in $h_{B}$.

Proof. Let $h_{A}$ be hesitant fuzzy semiopen in $X$, then there is a hesitant fuzzy open set $h_{U}$ such that $h_{U} \subseteq h_{A} \subseteq c_{H}\left(h_{U}\right)$ implies that $h_{U} \subseteq h_{A} \subseteq h_{B}$. Hence, 
$h_{U} \subseteq h_{A} \subseteq c l_{H}\left(h_{U}\right) \widetilde{\cap} h_{B}=c l_{\tau_{h_{B}}}\left(h_{U}\right)$. Since $h_{U} \widetilde{\cap} h_{B}=h_{U}$ is also hesitant fuzzy open in $h_{B}$, then $h_{A}$ is hesitant fuzzy semiopen in $h_{B}$.

Conversely, let $h_{A}$ be hesitant fuzzy semiopen in $h_{B}$. Then there is a hesitant fuzzy open set $h_{U}$ in $h_{B}$ such that $h_{U} \subseteq h_{A} \subseteq c l_{\tau_{h_{B}}}\left(h_{U}\right)$. Since $h_{U}$ is hesitant fuzzy open in $h_{B}$, there exists a hesitant fuzzy open set $h_{V}$ such that $h_{U}=h_{V} \widetilde{\cap} h_{B}$. Then, $h_{V} \widetilde{\cap} h_{B}=h_{U} \subseteq h_{A} \subseteq c l_{\tau_{h_{B}}}\left(h_{U}\right)=c l_{\tau_{h_{B}}}\left(h_{V} \widetilde{\cap} h_{B}\right) \subseteq c l_{H}\left(h_{V} \widetilde{\cap} h_{B}\right)$. By Theorem $4(2), h_{V} \widetilde{\cap} h_{B}$ is hesitant fuzzy semiopen, then by Theorem $11(2), h_{A}$ is hesitant fuzzy semiopen in $X$.

Theorem 14. Let $(X, \tau)$ be a hesitant fuzzy topological space, $h_{A}, h_{B} \in H S(X)$, $h_{A} \subseteq h_{B}$ and $h_{B}$ be hesitant fuzzy preopen in $X$. Then, $h_{A}$ is hesitant fuzzy preopen in $X$ if and only if $h_{A}$ is hesitant fuzzy preopen in $h_{B}$.

Proof. Suppose that $h_{A}$ is hesitant fuzzy preopen in $X$, then $h_{A}=h_{A} \widetilde{\cap} h_{B} \subseteq$ $\operatorname{int}_{H}\left(c l_{H}\left(h_{A}\right)\right) \widetilde{\cap} h_{B}$. Since $\operatorname{int}_{H}\left(c l_{H}\left(h_{A}\right)\right) \widetilde{\cap} h_{B}$ is hesitant fuzzy open in $h_{B}$, then $h_{A} \subseteq \operatorname{int}_{H}\left(c l_{H}\left(h_{A}\right)\right) \widetilde{n} h_{B} \subseteq \operatorname{int}_{\tau_{h_{B}}}\left[i n t_{H}\left(c l_{H}\left(h_{A}\right)\right) \widetilde{n} h_{B}\right] \subseteq i n t_{\tau_{h_{B}}}\left[c l_{H}\left(h_{A}\right) \widetilde{\cap} h_{B}\right]=$ int $_{\tau_{h_{B}}}\left(c l_{\tau_{h_{B}}}\left(h_{A}\right)\right)$. Hence, $h_{A}$ is hesitant fuzzy preopen in $h_{B}$.

Conversely, assume that $h_{A}$ is hesitant fuzzy preopen in $h_{B}$. Then, $h_{A} \subseteq$ $\operatorname{int}_{\tau_{h_{B}}}\left(c l_{\tau_{h_{B}}}\left(h_{A}\right)\right)$. Since $i n t_{\tau_{h_{B}}}\left(c l_{\tau_{h_{B}}}\left(h_{A}\right)\right)$ is hesitant fuzzy open in $h_{B}$, so there a hesitant fuzzy open set $h_{U}$ in $X$ such that $i n t_{\tau_{h_{B}}}\left(c l_{\tau_{h_{B}}}\left(h_{A}\right)\right)=h_{U} \widetilde{\cap} h_{B}$. By Theorem $9(2), i n t_{\tau_{h_{B}}}\left(c l_{\tau_{h_{B}}}\left(h_{A}\right)\right)$ is hesitant fuzzy preopen in $X$. Therefore,

$$
\begin{gathered}
h_{A} \subseteq \operatorname{int}_{\tau_{h_{B}}}\left(c l_{\tau_{h_{B}}}\left(h_{A}\right)\right) \\
\subseteq \operatorname{int}_{H}\left(c l_{H}\left(\operatorname{int}_{\tau_{h_{B}}}\left(c l_{\tau_{h_{B}}}\left(h_{A}\right)\right)\right)\right) \\
=\operatorname{int}_{H}\left(c l_{H}\left(\operatorname{int}_{\tau_{h_{B}}}\left[c l_{H}\left(h_{A}\right) \widetilde{\cap} h_{B}\right]\right)\right) \\
\subseteq \operatorname{int}_{H}\left(c l_{H}\left[c_{H}\left(h_{A}\right) \widetilde{\cap} h_{B}\right]\right) \\
\subseteq \operatorname{int}_{H}\left(c l_{H}\left(c l_{H}\left(h_{A}\right)\right)\right) \\
=\operatorname{int}_{H}\left(c l_{H}\left(h_{A}\right)\right) .
\end{gathered}
$$

This shows that $h_{A}$ is hesitant fuzzy preopen in $X$.

Declaration of Competing Interests The author of this paper declare that there are no conflicts of interest about publication of the paper.

\section{REFERENCES}

[1] Abd El-Monsef, M. E., El-Deeb, S. N., Mahmoud, R. A., $\beta$-open sets and $\beta$-continuous mappings, Bull. Fac. Sci. Assuit Univ., 12 (1983), 77-90.

[2] Andrijevic, D., On b-open sets, Mat. Vesnik, 48 (1996), 59-64.

[3] Chang, C. L., Fuzzy topological spaces, J. Math. Anal. Appl., 24(1) (1968), 182-190.

[4] Divakaran, D., John, S. J., Hesitant fuzzy rough sets through hesitant fuzzy relations, Ann. Fuzzy Math. Inform., 8 (2014), 33-46. 
[5] Jun, Y. B., Lee, K. J., Song, S. Z., Hesitant fuzzy bi-ideals in semigroups, Commun. Korean Math. Soc., 30(3) (2015), 143-154. http://dx.doi.org/10.4134/CKMS.2015.30.3.143

[6] Jun, Y. B., Ahn, S. S., Hesitant fuzzy sets theory applied to BCK/BCI-algebras, J. Comput. Anal. Appl., 20 (2016), 635-646.

[7] Kim, J., Lim, P. K., Lee, J. G., Hur, K., Hesitant fuzzy sets applied to BCK/BCI-algebras, Ann. Fuzzy Math. Inform., 18(3) (2019), 209-231. https://doi.org/10.30948/afmi.2019.18.3.209

[8] Kim, J., Jun, Y. B., Lim, P. K., Lee, J. G., Hur, K., The category of hesitant H-fuzzy sets, Ann. Fuzzy Math. Inform., 18(1) (2019), 57-74. https://doi.org/10.30948/afmi.2019.18.1.57

[9] Kim, J., Lim, P. K., Lee, J. G., Hur, K., Hesitant fuzzy subgroups and subrings, Ann. Fuzzy Math. Inform., 18(2) (2019), 105-122. https://doi.org/10.30948/afmi.2019.18.2.105

[10] Lee, J.-G., Hur, K., Hesitant fuzzy topological spaces, Mathematics, 8(2) (2020), 1-21. https://doi.org/10.3390/math8020188

[11] Levine, N., Semi-open sets and semi-continuity in topological spaces, Amer. Math. Monthly, 70 (1) (1963), 34-41.

[12] Mashhour, A. S., Abd El-Monsef, M. E., El-Deeb, S. N., On precontinuous and weak precontinuous functions, Proc. Math. Phys. Soc. Egypt, 51 (1982), 47-53.

[13] Njstad, O., On some classes of nearly open sets, Pacific J. Math., 15 (1965), 961-970.

[14] Torra, V., Hesitant fuzzy sets, Int. J. Intell. Syst., 25(6) (2010), 529-539. https://doi.org/10.1002/int.20418

[15] Xia, M., Xu, Z., Hesitant fuzzy information aggregation in decision making, Int. J. Approx. Reason., 52(3) (2011), 395-407. https://doi.org/10.1016/j.ijar.2010.09.002

[16] Zadeh, L. A., Fuzzy sets, Inf. Control., 8(3) (1965), 338-353. 